\title{
Cluster Based Multifunctional Copper(II) Organic Framework as Photocatalyst in the Degradation of Organic Dye and Electrocatalyst for Overall Water Splitting
}

Feng Wang, Fengkun Tian, Yuxin Deng, Lulu Yang, Huihui Zhang, Dongsheng Zhao, Bei Li, Xiutang Zhang," and Liming Fan*

5 Department of Chemistry, College of Science, North University of China, Taiyuan 030051, P. R. China.

E-mail: limingfan@nuc.edu.cn; xiutangzhang@163.com.

Comment S1 Materials and characterization for 1 2

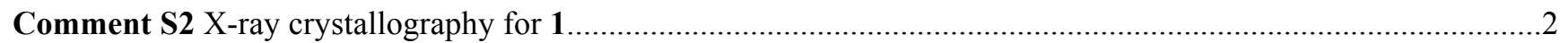

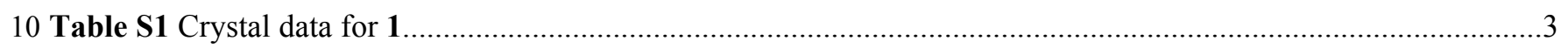

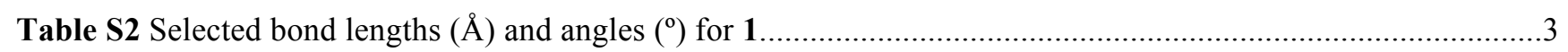

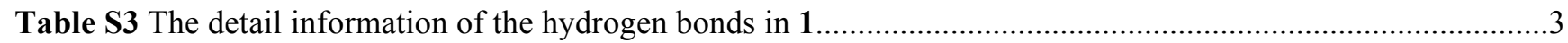

Table S4 Comparison of electrocatalytic performances of MOFs based materials for OER......................................4

Table S5 Comparison of electrocatalytic performances of MOFs based materials for HER....................................

15 Scheme S1 The ligand of 1-(tetrazo-5-yl)-4-(triazo-1-yl) benzene (HTTB) in the construction of 1........................4

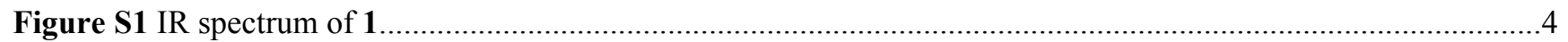

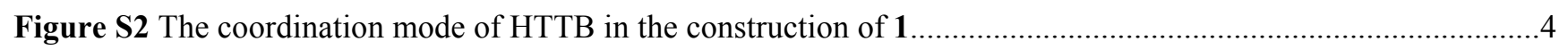

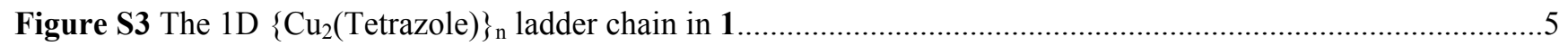

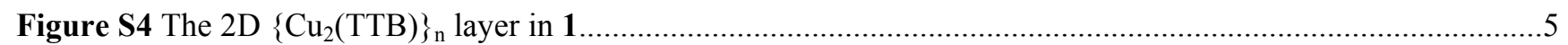

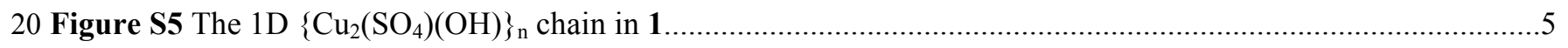

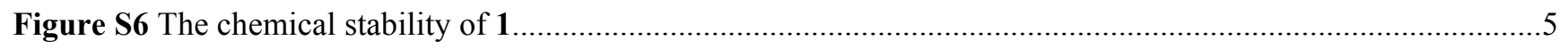

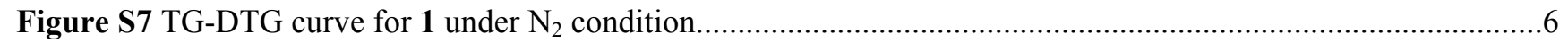

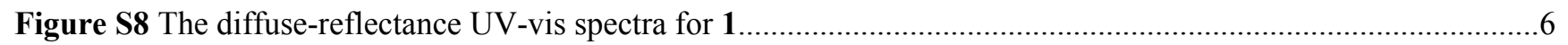

Figure S9 Kubelka-Munk-transformed diffuse reflectance for 1 ................................................................6

25 Figure S10 Recycling experiments of photocatalytic degradation of MB by 1 ..................................................

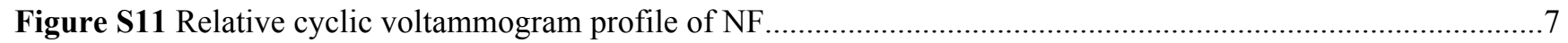

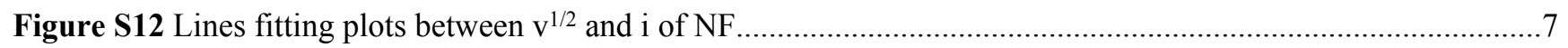

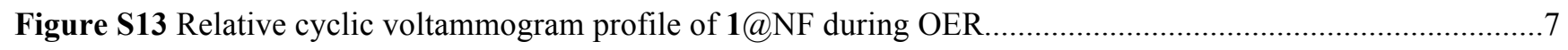

Figure S14 Relative cyclic voltammogram profile of $\mathrm{RuO}_{2} @ \mathrm{NF}$ during OER ....................................................... 8

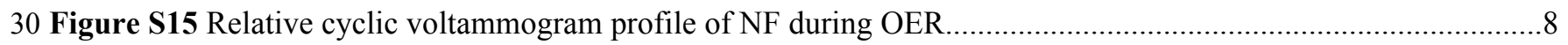

Figure S16 Relative cyclic voltammogram profile of 1@NF during HER ….......................................................

Figure S17 Relative cyclic voltammogram profile of Pt/C@NF during HER ..........................................................

Figure S18 Relative cyclic voltammogram profile of NF during HER ................................................................. 


\section{Comment S1: Materials and characterization for 1.}

All the chemical reagents were commercially purchased without further purification. IR spectra were measured on a NEXUS 670 FTIR spectrometer. Thermogravimetric analyses (TGA) were heat from room temperature to $800{ }^{\circ} \mathrm{C}$ at $10^{\circ} \mathrm{C}$ /min under $\mathrm{N}_{2}$ condition on the PerkinElmer DTA 6000 thermogravimetric analyzer. The electrochemical measurements 5 were carried out at room temperature on a CHI660E of chenhua electrochemical workstation in shanghai. X-ray diffraction (XRD) data were measured on a Rigaku D-MAX 2500/PC using Cu K $\alpha$ radiation $(\lambda=1.54056 \AA$ ). Elemental analyses including $\mathrm{C}, \mathrm{H}, \mathrm{N}$ were measured using Vario MACRO cube elemental analyzer. UV-vis absorption spectra were recorded on Shimadzu UV-2600 spectrophotometer.

\section{Comment S2: X-ray crystallography for 1.}

Structural integrity single crystal of $\mathbf{1}$ was carefully selected under an optical microscope and fixed to thin glass fibers. After that, single-crystal X-ray diffraction analyses was performed on a Siemens SMART diffractometer using Mo-K $\alpha$ radiation $(\lambda=0.71073 \AA$ ). The structure of 1 was solved by direct methods, with the non-hydrogen atoms refined anisotropially by using the SHELXTL-2016 package with $F^{2}$ values based full-matrix least-squares procedure and Olex 2.0., ${ }^{1,2}$ All the 15 hydrogen atoms except those for water molecules were generated geometrically with fixed isotropic thermal parameters, and included in the structure factor calculations. And the hydrogen atoms attached to oxygen were refined with $\mathrm{O}-\mathrm{H}=0.85 \AA$ and $\mathrm{U}_{\text {iso }}(\mathrm{H})=1.2 \mathrm{U}_{\text {eq }}(\mathrm{O})$. The crystallographic data, the details of the crystal structures for $\mathbf{1}$ are listed in Table S1. Selected bond lengths and angles for $\mathbf{1}$ are shown in Table S2. The CCDC number is 2061617. 
Table S1 Crystal data for $\mathbf{1}$.

\begin{tabular}{ll}
\hline Formula & $\mathrm{C}_{11} \mathrm{H}_{14} \mathrm{Cu}_{2} \mathrm{~N}_{8} \mathrm{O}_{7} \mathrm{~S}$ \\
Formula weight & 529.46 \\
Crystal system & Triclinic \\
Space group & $P-1$ \\
$a(\AA)$ & $6.9971(2)$ \\
$b(\AA)$ & $8.1761(3)$ \\
$c(\AA)$ & $15.7689(5)$ \\
$\alpha\left({ }^{\circ}\right)$ & $78.879(3)$ \\
$\beta\left({ }^{\circ}\right)$ & $86.107(2)$ \\
$\gamma\left({ }^{\circ}\right)$ & $78.789(2)$ \\
$V\left(\AA^{3}\right)$ & $867.86(5)$ \\
$Z$ & 2 \\
$D_{\text {calcd }}\left(\mathrm{Mg} / \mathrm{m}^{3}\right)$ & 2.026 \\
$\left.\mu(\mathrm{mm})^{-1}\right)$ & 4.706 \\
Temperature $(\mathrm{K})$ & $150 \mathrm{~K}$ \\
$F(000)$ & 532.0 \\
$R_{\text {int }}$ & 0.0212 \\
$R_{1}[I>2 \sigma(I)]^{\mathrm{a}}$ & 0.0288 \\
$\mathrm{wR}_{2}[I>2 \sigma(I)]^{\mathrm{b}}$ & 0.0813 \\
$\mathrm{Gof}$ & 1.075 \\
a) $\left.R_{1}=\Sigma|| F_{\mathrm{o}}|-| F_{\mathrm{c}}|\Sigma|\left|F_{\mathrm{o}}\right| \cdot \mathrm{b}\right) w R_{2}=\left\{\left[\Sigma w\left(F_{\mathrm{o}}^{2}-F_{\mathrm{c}}^{2}\right)^{2} / \Sigma w\left(F_{\mathrm{o}}^{2}\right)^{2}\right]\right\}^{1 / 2}$. & \\
\hline
\end{tabular}

5 Table S2 Selected bond lengths $(\AA)$ and angles $\left({ }^{\circ}\right)$ for $\mathbf{1}$.

\begin{tabular}{|c|c|c|c|c|c|c|c|}
\hline$\overline{\mathrm{Cu} 1-\mathrm{O} 1}$ & $1.9886(15)$ & Cu3-N4 & $2.0003(19)$ & $\mathrm{O}^{\# 4}-\mathrm{Cu} 2-\mathrm{O}^{\# 5}$ & $89.08(6)$ & $\mathrm{O}^{\# 1}-\mathrm{Cu} 1-\mathrm{N} 1^{\# 3}$ & $89.67(7)$ \\
\hline $\mathrm{Cu} 1-\mathrm{O} 1^{\# 1}$ & $1.9886(15)$ & $\mathrm{Cu} 3-\mathrm{N6}^{\# 4}$ & $2.4514(19)$ & $\mathrm{O}^{\# 5}-\mathrm{Cu} 2-\mathrm{O}^{\# 4}$ & 180.0 & $\mathrm{O} 1-\mathrm{Cu} 1-\mathrm{N} 1^{\# 3}$ & $90.33(7)$ \\
\hline $\mathrm{Cu} 1-\mathrm{O} 2$ & $2.3178(17)$ & $\mathrm{O} 1-\mathrm{Cu} 1-\mathrm{O} 1^{\# 1}$ & 180.0 & $\mathrm{~N} 2^{\# 6}-\mathrm{Cu} 2-\mathrm{O} 1^{\# 4}$ & $92.63(6)$ & $\mathrm{O} 2-\mathrm{Cu} 1-\mathrm{O} 2^{\# 1}$ & $180.00(5)$ \\
\hline $\mathrm{Cu} 1-\mathrm{O} 2^{\# 1}$ & $2.3179(17)$ & $\mathrm{O} 1-\mathrm{Cu} 1-\mathrm{O} 2$ & $89.21(6)$ & $\mathrm{N} 2-\mathrm{Cu} 2-\mathrm{O} 1^{\# 4}$ & $87.37(6)$ & $\mathrm{O} 5-\mathrm{Cu} 3-\mathrm{N6}^{\# 5}$ & $91.26(7)$ \\
\hline $\mathrm{Cu} 1-\mathrm{N} 1{ }^{\# 2}$ & $2.0252(18)$ & $\mathrm{O} 1-\mathrm{Cu} 1-\mathrm{O} 22^{\# 1}$ & $90.79(6)$ & $\mathrm{N} 2{ }^{\# 6}-\mathrm{Cu} 2-\mathrm{O} 1^{\# 5}$ & $87.37(6)$ & $\mathrm{N} 3^{\# 2}-\mathrm{Cu} 3-\mathrm{N} 6^{5 \#}$ & $177.42(7)$ \\
\hline $\mathrm{Cu} 1-\mathrm{N} 1{ }^{\# 3}$ & $2.0252(18)$ & $\mathrm{O}^{\# 1}-\mathrm{Cu} 1-\mathrm{O} 2$ & $90.80(6)$ & N2-Cu2-O1 $\# 5$ & $92.63(6)$ & N4-Cu3-O4 & $85.89(8)$ \\
\hline $\mathrm{Cu} 2-\mathrm{O} 1^{\# 4}$ & $2.3009(15)$ & $\mathrm{O} 1^{\# 1}-\mathrm{Cu} 1-\mathrm{O} 2^{\# 1}$ & $89.21(6)$ & $\mathrm{N} 2^{\# 6}-\mathrm{Cu} 2-\mathrm{O}^{\# 4}$ & $90.50(7)$ & N4-Cu3-O5 & $92.38(8)$ \\
\hline $\mathrm{Cu} 2-\mathrm{O} 1^{\# 5}$ & $2.3009(15)$ & $\mathrm{N} 1^{\# 3}-\mathrm{Cu} 1-\mathrm{O} 2^{\# 1}$ & $92.80(7)$ & N2-Cu2-O3 ${ }^{\# 5}$ & $90.50(7)$ & O1-Cu3-N4 & $175.43(8)$ \\
\hline $\mathrm{Cu} 2-\mathrm{O}^{\# 5}$ & $2.0273(17)$ & $\mathrm{N} 1{ }^{\# 2}-\mathrm{Cu} 1-\mathrm{O} 2$ & $92.80(7)$ & $\mathrm{N} 2-\mathrm{Cu} 2-\mathrm{O}^{\# 4}$ & $89.50(7)$ & $\mathrm{O} 1-\mathrm{Cu} 3-\mathrm{N6}^{\# 5}$ & $91.12(6)$ \\
\hline $\mathrm{Cu} 2-\mathrm{O}^{\# 4}$ & $2.0273(17)$ & $\mathrm{N} 1^{\# 2}-\mathrm{Cu} 1-\mathrm{O} 2^{\# 1}$ & $87.20(7)$ & $\mathrm{N} 2{ }^{\# 6}-\mathrm{Cu} 2-\mathrm{O}^{\# 5}$ & $89.50(7)$ & $\mathrm{O} 4-\mathrm{Cu} 3-\mathrm{O} 5$ & $178.27(7)$ \\
\hline $\mathrm{Cu} 2-\mathrm{N} 2{ }^{\# 6}$ & $1.9844(18)$ & $\mathrm{N} 1{ }^{\# 3}-\mathrm{Cu} 1-\mathrm{O} 2$ & $87.20(7)$ & $\mathrm{N} 2-\mathrm{Cu} 2-\mathrm{N} 22^{\# 6}$ & 180.0 & $\mathrm{O} 4-\mathrm{Cu} 3-\mathrm{N} 33^{\# 2}$ & $92.89(7)$ \\
\hline $\mathrm{Cu} 2-\mathrm{N} 2$ & $1.9844(19)$ & $\mathrm{N} 1^{\# 2-C u 1-N 1} 1^{\# 3}$ & 180.0 & $\mathrm{O} 1-\mathrm{Cu} 3-\mathrm{O} 4$ & $89.95(7)$ & $\mathrm{O} 4-\mathrm{Cu} 3-\mathrm{N}^{\# 5}$ & $88.80(7)$ \\
\hline $\mathrm{Cu} 3-\mathrm{O} 1$ & $1.9583(15)$ & 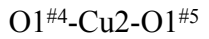 & $180.00(7)$ & $\mathrm{O} 1-\mathrm{Cu} 3-\mathrm{O} 5$ & $91.78(7)$ & $\mathrm{O} 5-\mathrm{Cu} 3-\mathrm{N} 33^{\# 2}$ & $87.11(7)$ \\
\hline $\mathrm{Cu} 3-\mathrm{O} 4$ & $2.0337(18)$ & $\mathrm{O}^{\# 5}-\mathrm{Cu} 2-\mathrm{O} 1^{\# 5}$ & $90.92(6)$ & $\mathrm{O} 1-\mathrm{Cu} 3-\mathrm{N}^{\# 2}$ & $86.94(6)$ & $\mathrm{N} 4-\mathrm{Cu} 3-\mathrm{N} 33^{\# 2}$ & $95.18(7)$ \\
\hline $\mathrm{Cu} 3-\mathrm{O} 5$ & $2.0395(18)$ & $\mathrm{O}^{\# 5}-\mathrm{Cu} 2-\mathrm{O} 1^{\# 4}$ & $89.08(6)$ & $\mathrm{O} 1-\mathrm{Cu} 1-\mathrm{N} 1^{\# 2}$ & $89.67(7)$ & $\mathrm{N} 4-\mathrm{Cu} 3-\mathrm{N}^{5 \#}$ & $86.88(7)$ \\
\hline $\mathrm{Cu} 3-\mathrm{N} 3{ }^{\# 2}$ & $2.3570(19)$ & $\mathrm{O}^{\# 4}-\mathrm{Cu} 2-\mathrm{O}^{\# 4}$ & $90.92(6)$ & $\mathrm{O} 1^{\# 1}-\mathrm{Cu} 1-\mathrm{N} 1^{\# 2}$ & $90.33(7)$ & & \\
\hline
\end{tabular}

Table S3 The detail information of the hydrogen bonds in $\mathbf{1}$.

\begin{tabular}{|c|c|c|c|}
\hline$\overline{\mathrm{D}-\mathrm{H} \cdots \mathrm{A}}$ & $\mathrm{H} \cdots \mathrm{A}(\AA)$ & $\mathrm{D} \cdots \mathrm{A}(\AA)$ & $\angle \mathrm{D}-\mathrm{H} \cdots \mathrm{A}\left({ }^{\circ}\right)$ \\
\hline $\mathrm{O} 5-\mathrm{H} 5 \mathrm{~A} \cdots \mathrm{O} 6^{\mathrm{i}}$ & 1.93 & $2.744(3)$ & 160 \\
\hline $\mathrm{O} 5-\mathrm{H} 5 \mathrm{~A} \cdots \mathrm{O} 7$ & 1.92 & $2.761(4)$ & 168 \\
\hline $\mathrm{O} 7-\mathrm{H} 7 \mathrm{~A} \cdots \mathrm{O} 2^{\mathrm{ii}}$ & 1.97 & $2.809(4)$ & 172 \\
\hline $\mathrm{O} 7-\mathrm{H} 7 \mathrm{~B} \cdots \mathrm{O} 3^{\mathrm{iii}}$ & 2.19 & $2.970(3)$ & 152 \\
\hline $\mathrm{C} 4-\mathrm{H} 4 \cdots \mathrm{N} 8$ & 2.45 & $3.381(5)$ & 166 \\
\hline C6-H6№ & 2.45 & $3.374(5)$ & 163 \\
\hline $\mathrm{C} 7-\mathrm{H} 7 \cdots \mathrm{O}^{\mathrm{iv}}$ & 2.24 & $3.182(3)$ & 173 \\
\hline \multicolumn{4}{|c|}{ Symmetry codes: i $x,-1+y, z$; ii $1-x, 2-y,-z$; iii $1+x,-1+y, z$; iv $-x, 2-y, 1-z$. } \\
\hline
\end{tabular}


Table. S4 Comparison of electrocatalytic performances of MOFs based materials for OER.

\begin{tabular}{|c|c|c|c|c|}
\hline Catalyst & Substrate & Electrolyte & $\eta_{10}$ & Reference \\
\hline $\mathrm{Co}-\mathrm{BPDC} / \mathrm{Co}-\mathrm{BDC}_{3}$ & GCE & $1 \mathrm{M} \mathrm{KOH}$ & $335 \mathrm{mV}$ & 48 \\
\hline UTSA-16 & GCE & $1 \mathrm{M} \mathrm{KOH}$ & $408 \mathrm{mV}$ & 49 \\
\hline Co-ZIF-9(III) & GCE & $1 \mathrm{M} \mathrm{KOH}$ & $380 \mathrm{mV}$ & 50 \\
\hline Unsaturated ZIF-67 & GCE & $1 \mathrm{M} \mathrm{KOH}$ & $410 \mathrm{mV}$ & 51 \\
\hline CTGU-14 & GCE & $1 \mathrm{M} \mathrm{KOH}$ & $454 \mathrm{mV}$ & 52 \\
\hline 1@NF & NF & $1 \mathrm{M} \mathrm{KOH}$ & $377 \mathrm{mV}$ & this work \\
\hline Catalyst & Substrate & Electrolyte & $\eta_{10}$ & Reference \\
\hline $\mathrm{Ni}-\mathrm{ZIF}$ & GCE & $1 \mathrm{M} \mathrm{KOH}$ & $218 \mathrm{mV}$ & 54 \\
\hline $\mathrm{Fe}_{2} \mathrm{Co}-\mathrm{MOF}$ & GCE & $1 \mathrm{M} \mathrm{KOH}$ & $221 \mathrm{mV}$ & 55 \\
\hline $\mathrm{NiFe}-\mathrm{MOF}$ & NF & $1 \mathrm{M} \mathrm{KOH}$ & $255 \mathrm{mV}$ & 56 \\
\hline $\mathrm{Co} / \mathrm{Cu}-\mathrm{MOF}$ & GCE & $1 \mathrm{M} \mathrm{KOH}$ & $391 \mathrm{mV}$ & 57 \\
\hline $\mathrm{Ni}-\mathrm{CP}$ & GCE & $1 \mathrm{M} \mathrm{KOH}$ & $422 \mathrm{mV}$ & 58 \\
\hline 1@NF & NF & $1 \mathrm{M} \mathrm{KOH}$ & $242 \mathrm{mV}$ & this work \\
\hline
\end{tabular}

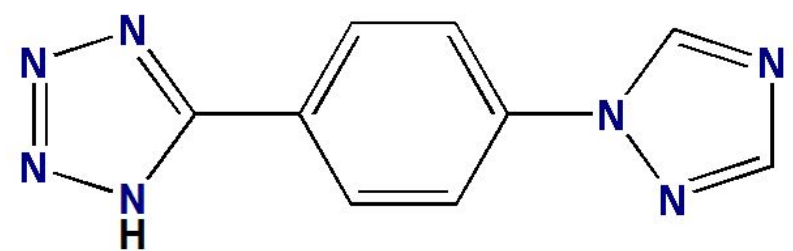

Scheme S1. The ligand of 1-(tetrazo-5-yl)-4-(triazo-1-yl) benzene (HTTB) in the construction of 1.

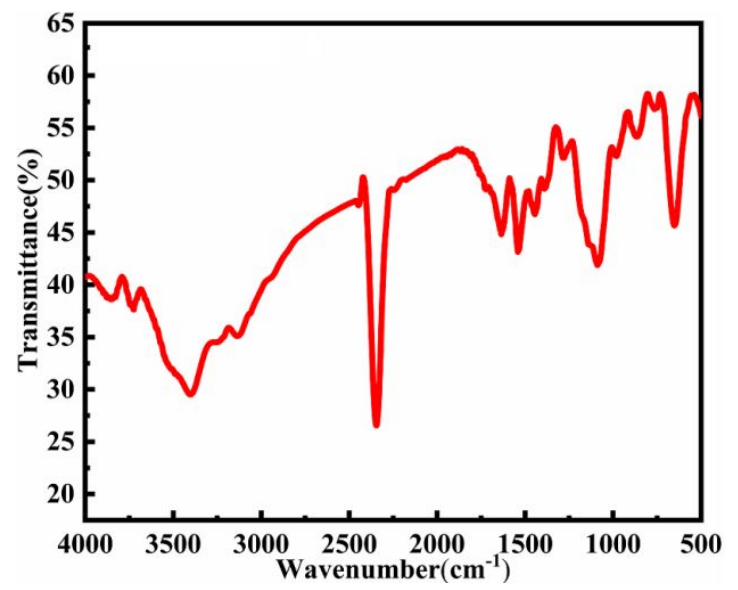

Figure S1. IR spectrum of $\mathbf{1}$.

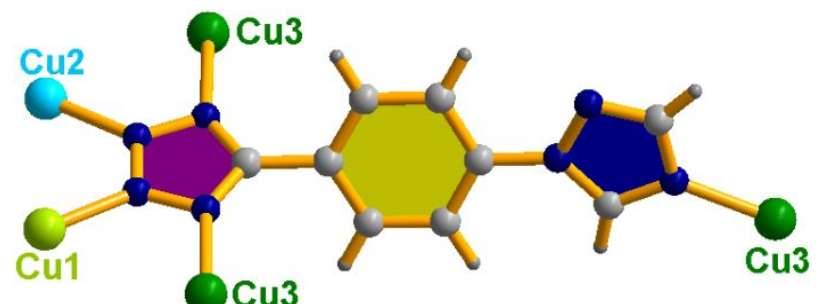

Figure S2. The coordination mode of HTTB in the construction of $\mathbf{1 .}$ 


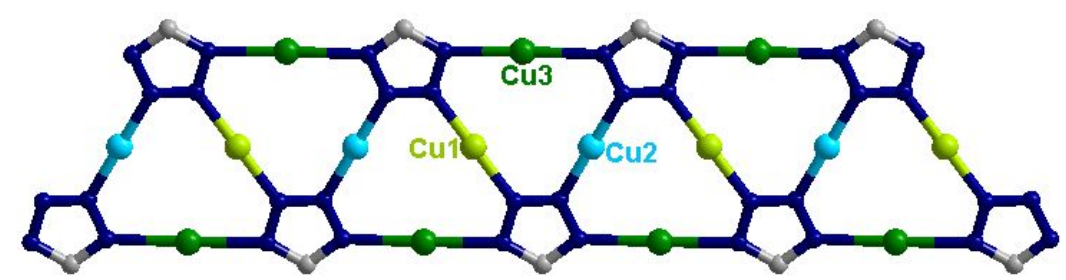

Figure S3. The 1D $\left\{\mathrm{Cu}_{2} \text { (Tetrazole) }\right\}_{\mathrm{n}}$ ladder chain in $\mathbf{1}$.

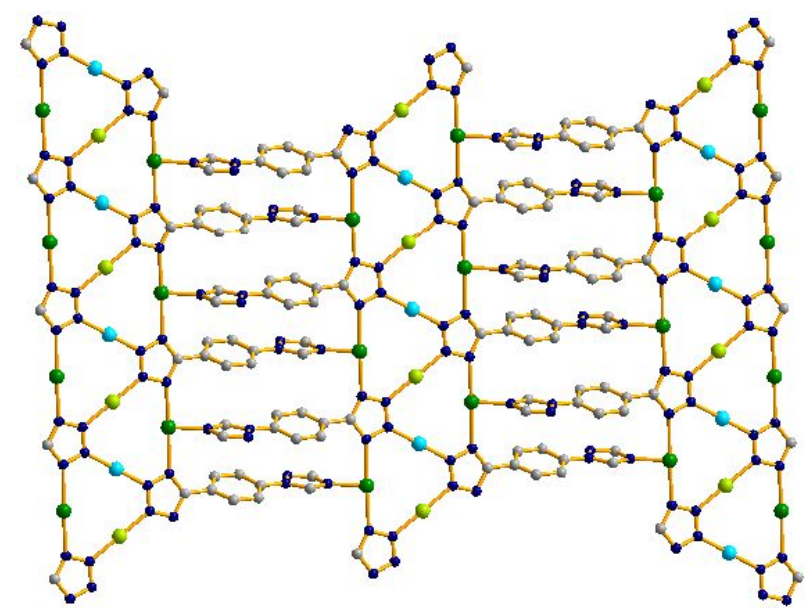

Figure S4. The 2D $\left\{\mathrm{Cu}_{2}(\mathrm{TTB})\right\}_{\mathrm{n}}$ layer in $\mathbf{1}$.

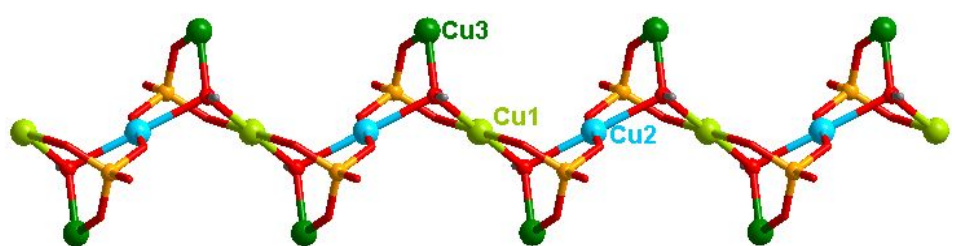

Figure S5. The 1D $\left\{\mathrm{Cu}_{2}\left(\mathrm{SO}_{4}\right)(\mathrm{OH})\right\}_{\mathrm{n}}$ chain in $\mathbf{1}$.

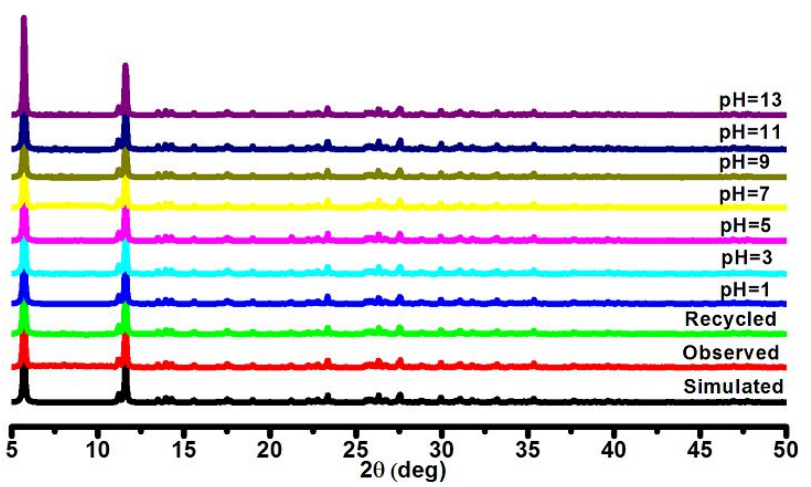

Figure S6. The chemical stability of $\mathbf{1}$. 


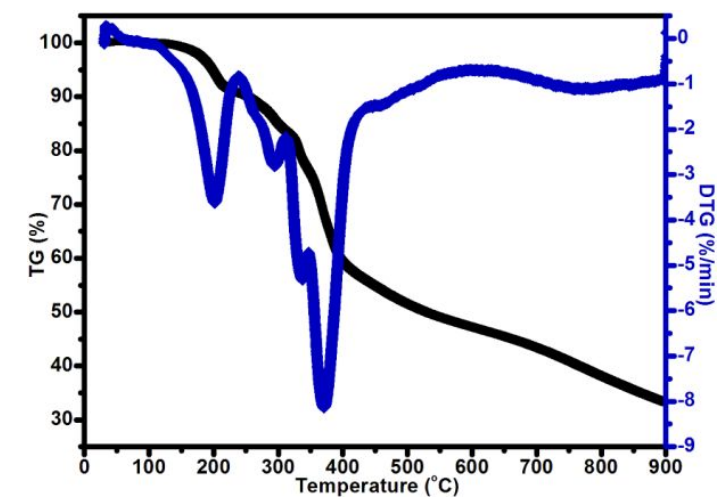

Figure S7. TG-DTG curve for 1 under $\mathrm{N}_{2}$ condition.

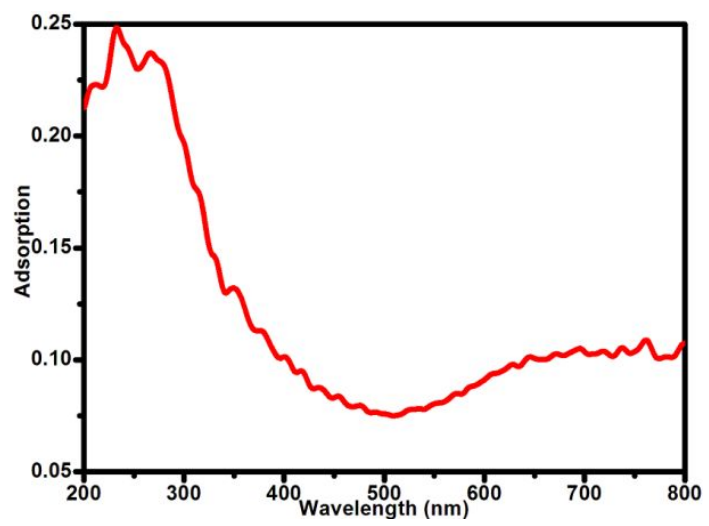

Figure S8. The diffuse-reflectance UV-vis spectra for 1.

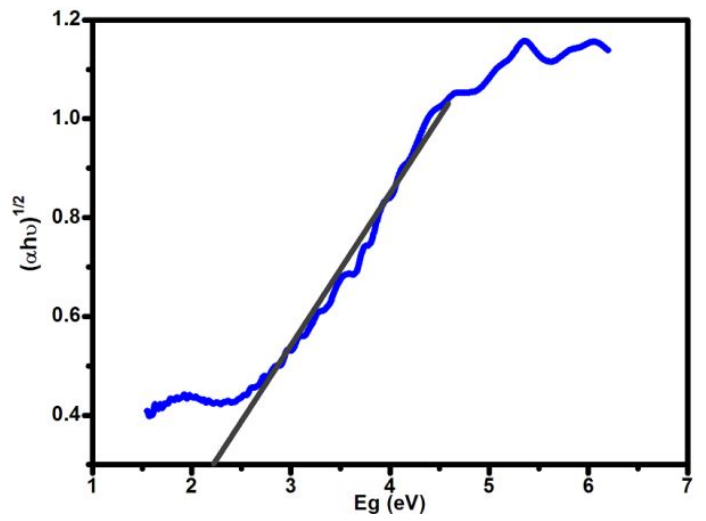

Figure S9. Kubelka-Munk-transformed diffuse reflectance for 1 .

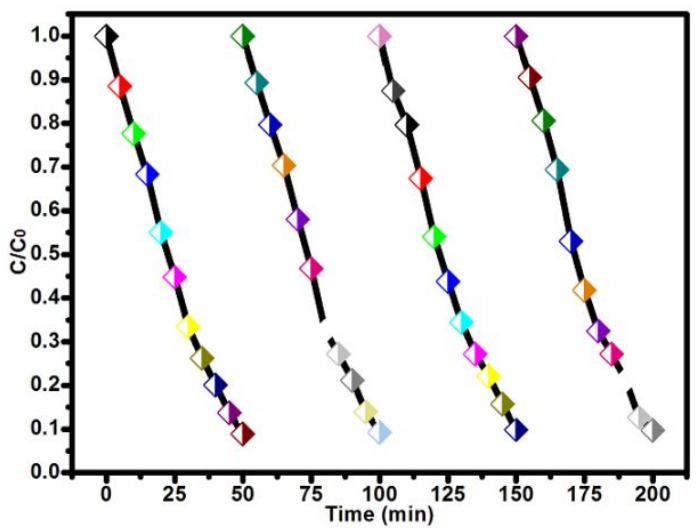

Figure S10. Recycling experiments of photocatalytic degradation of MB by $\mathbf{1}$. 


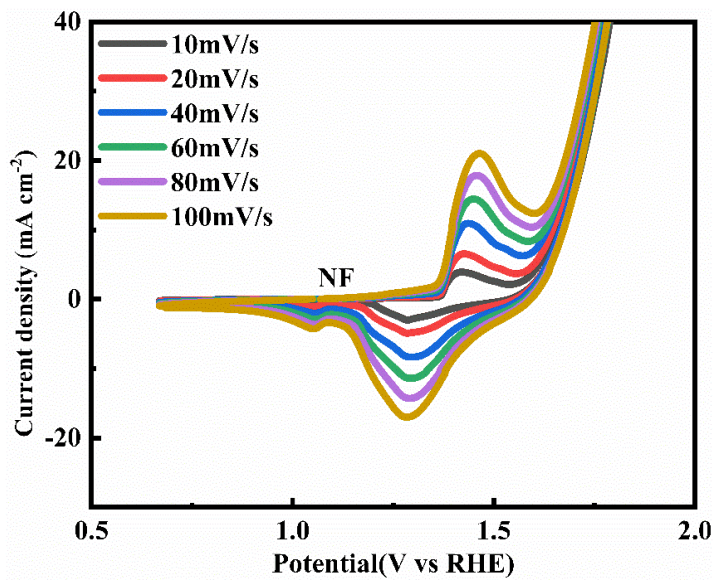

Figure S11. Relative cyclic voltammogram profile of NF.

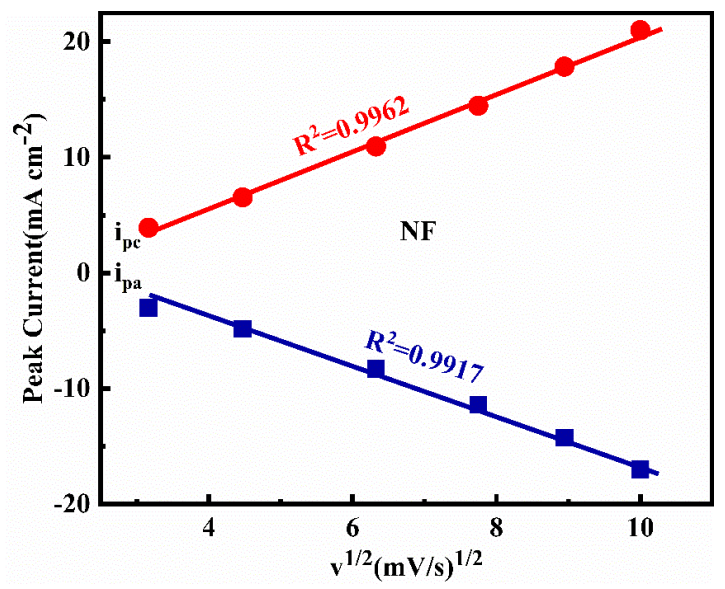

Figure S12. Lines fitting plots between $v^{1 / 2}$ and i of NF.

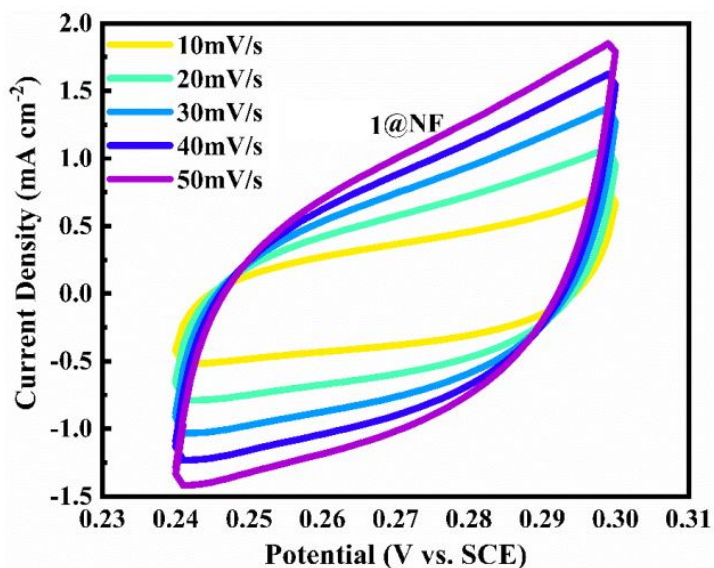

Figure S13. Relative cyclic voltammogram profile of $1 @ N F$ during OER. 


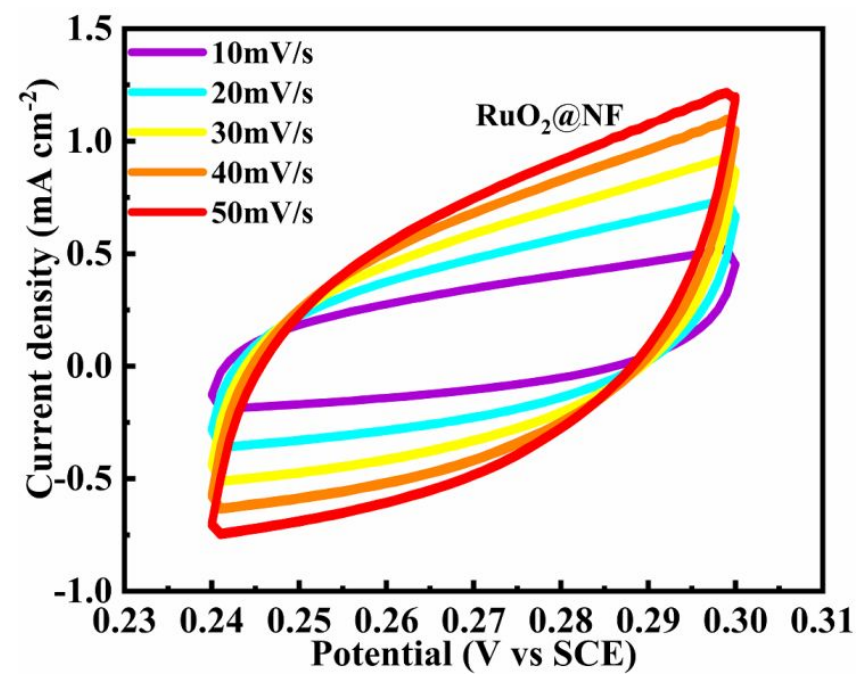

Figure S14. Relative cyclic voltammogram profile of $\mathrm{RuO}_{2} @ \mathrm{NF}$ during OER.

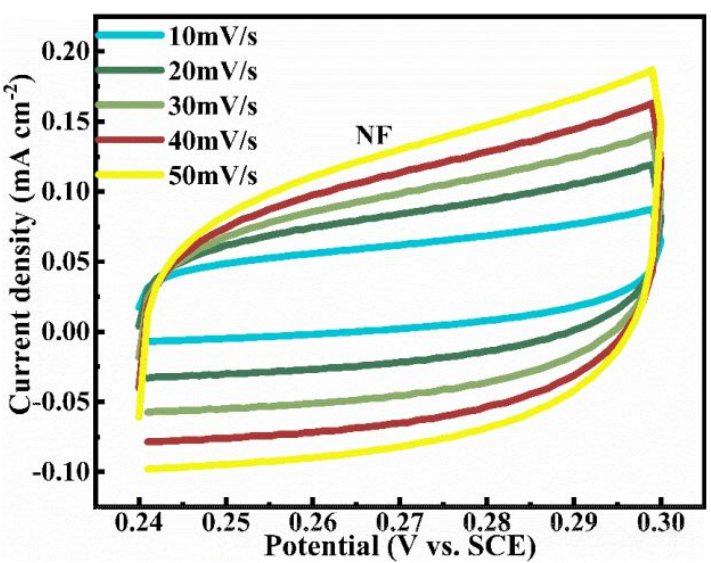

Figure S15. Relative cyclic voltammogram profile of NF during OER.

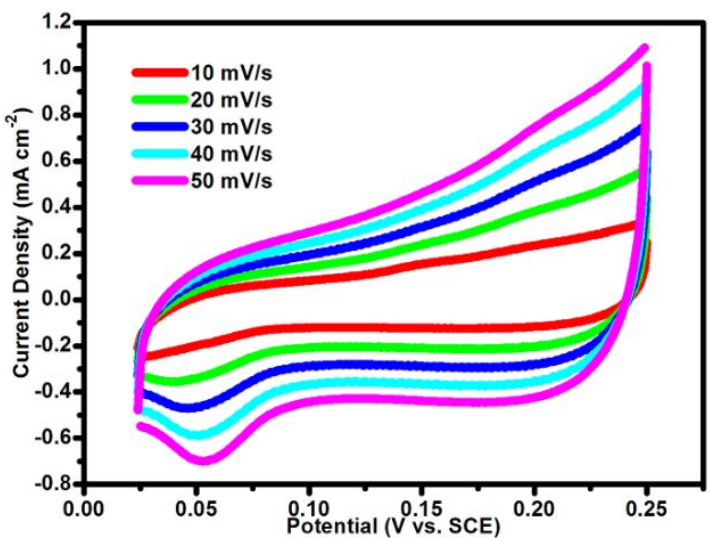

Figure S16. Relative cyclic voltammogram profile of $1 @$ NF during HER. 


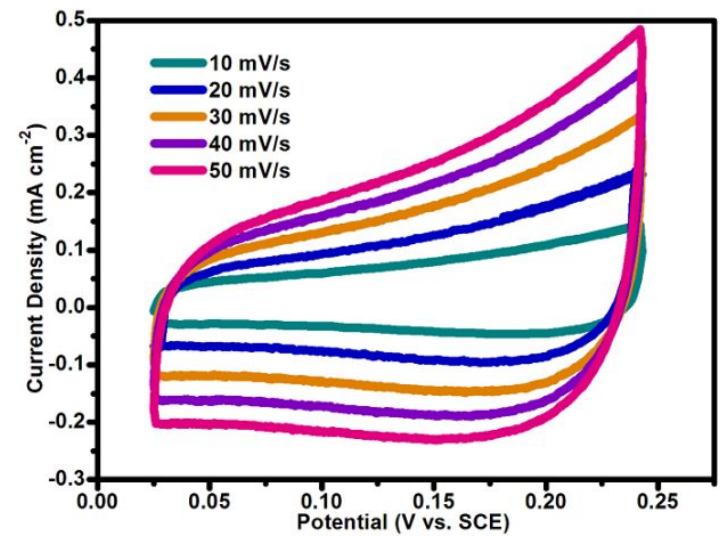

Figure S17. Relative Cyclic Voltammogram profile of Pt/C@NF during HER.

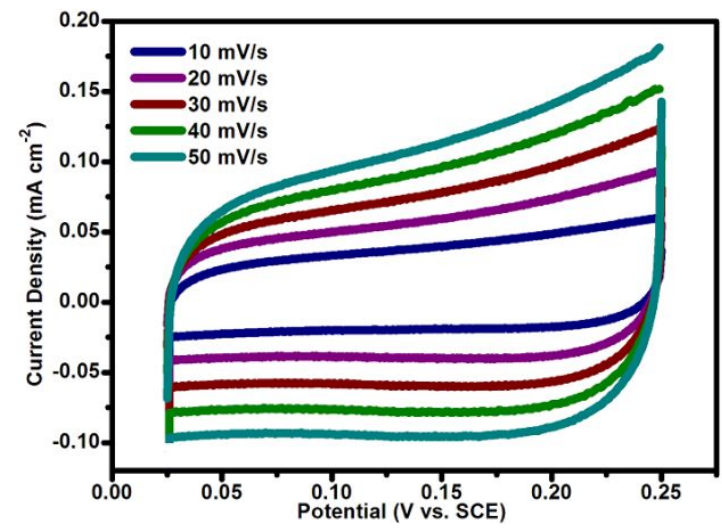

Figure S18. Relative cyclic voltammogram profile of NF during HER.

\section{Reference}

(1) Sheldrick, G. M. Crystal structure refinement with SHELXL. Acta Cryst. C 2015, 71, 3-8.

(2) Dolomanov, O. V.; Bourhis, L. J.; Gildea, R. J.; Howard, J. A. K.; Puschmann, H. OLEX2: a complete structure solution, refinement and analysis program. J. Appl. Crystallogr. 2009, 42, 339-341. 